\title{
On the Optimal Size of a Buffer Stock - The Case of Wheat in Pakistan
}

\author{
Peter A. CORNelisse and BART KuIJPERS*
}

\section{INTRODUCTION}

In a study of the operation of the wheat market in Pakistan, Cornelisse and Naqvi [1, p. 116] stated that the pre-harvest wheat stock in March 1983 of $2,100,000$ tons should provide sufficient protection against a major harvest failure. This conclusion was based on the observation that a stock of that size corresponded to approximately 40 percent of the marketed surplus of wheat and to 17 percent of domestic production - very secure proportions according to the prevailing standards.

Two questions, however, remain. One, it is not yet clear what the chances are that a bad harvest or a series of bad harvests wipes out the available stock and if that happens - what is the expected volume of wheat imports needed to supplement the stock. Two, while security is one concern in buffer stock management, the cost aspect is another. A large buffer stock provides excellent security, but it also entails high costs of storage; a smaller stock may reduce security only slightly and reduce costs considerably. On the other hand, if a buffer stock is small, the probability that supplementary imports are needed is relatively high and so is the expected volume of these imports. Considering the fact that in Pakistan domestically produced wheat is much cheaper than imported wheat, this implies that a small stock of wheat, too, may involve high costs, in this case due to imports. Thus the question arises: what is the size of the wheat buffer stock that minimizes costs?

Among other things, the present paper aims at providing answers to these questions. The argument is built up in three steps, each presented in a separate section. The next section specifies, by means of a simple model, the functions of a buffer stock in relation to other relevant variables. Thereafter, Section 3 deals with the probabilities of transient production deficits in excess of varying sizes of stocks and also with the expected volumes of supplementary imports that correspond with

*Dr Cornelisse is Professor at the Erasmus University, Rotterdam, while Mr. Kuijpers is Assistant to Professor Cornelisse. The authors wish to thank Maarten de Zeeuw for his help in an early stage of the research. 
these probabilities. The argument is illustrated empirically by the case of wheat in Pakistan. Section 4 presents estimates of expected recurrent costs involved by different sizes of buffer stocks. These estimates can serve as stepping stones to a decision on the optimal size of the stock. Finally, Section 5 contains some concluding remarks.

\section{FUNCTIONS AND PROPERTIES OF BUFFER STOCKS}

The balance equation of uses and availabilities of wheat can be written as

$$
V_{t}+M_{t+1}=C_{t+1}+\Delta B_{t+1} \cdots
$$

where

$V_{t}=$ domestic production of wheat in harvest year $t$,

$M_{t}^{t}=$ balance of imports and exports of wheat in harvest year $t$,

$C_{t}=$ consumption and losses of wheat in harvest year $t$, and

$\Delta B_{t}=$ change in the buffer stock of wheat in harvest year $t$. (It is assumed here that the stock is maintained only to compensate for fluctuations in the sizes of harvests over time.)

The growth of wheat consumption normally follows a smooth pattern. On the other hand, while domestic production of wheat, viewed over a longer period of time, may display structural growth, the fluctuations from year to year are erratic. Thus, variable $V$ can be divided into a structural component $(V S)$ and a transient component $(V T)$, where the buffer stock is meant to even out the latter. But the buffer stock can be supported in this respect by increases (decreases) of imports (exports) in the case of a bad harvest. Therefore, $M$, too, can be divided into a structural $(M S)$ and a transient $(M T)$ part. As a result, equation (1) can be decomposed into a structural balance equation

$$
V S_{t}+M S_{t+1}=C_{t+1}
$$

and a transient balance equation

$$
V T_{t}=\Delta B_{t+1}-M T_{t+1} \quad \ldots
$$

Note that if $V S>C$, the country concerned is a wheat exporter, whereas it is an importer if $V S<C$.

In this paper we concentrate on equation (3), as it links up with the buffer stock $(B)$. In fact, we are especially interested in those cases where $V T<0$, because they represent years of bad harvests, when the buffer stock, possibly supported by transient imports, is drawn upon. In these years $\Delta B<0$, where the extent to which a decrease in the stock, or a series of such decreases, can absorb fluctuations in the size of the harvest is obviously constrained by the size of the initial stock.

The simple constraint $B \geqslant 0$ implies, of course, that decreases in the stock are constrained, while increases - at least, in principle - are not. This introduces an asymmetry which is of considerable importance for a good understanding of equation (3). For ease of exposition, let us consider a country which has neither structural exports nor structural imports, so that the difference between production and consumption of wheat can directly be identified as the transient component of production. Assume further that this country possesses in the initial year a certain stock which is considered just right; thereafter it allows $V T$ to add to the stock in good years and reduce it in bad years. But when a particularly bad harvest eliminates the entire stock, supplementary wheat must be obtained from abroad. In other words, positive values of $V T$ are translated into equally large increases in the buffer stock, but negative values of $V T$ can, if measured in absolute terms, be larger than the accompanying decreases in the stock.

Hence, if the sum of values of $V T$ over an extended period of time is zero, the average value of increases and decreases in the stock must be positive. As a result, the actual average size of the stock will be larger than the desirable, initial stock. ${ }^{1}$ The difference, however, is significant only if the initial stock is so small that supplementary imports are frequent and relatively large. The above reasoning further shows that under these conditions the expected value of $M T$ in equation (3) is positive.

The type of buffer-stock regime as described above is assumed to prevail in the case examined in this paper. It must be underlined, however, that other types of management may be adopted. For example, the buffer stock may be subjected to an upper limit, corresponding to storage capacity. In that case, not only decreases but also increases in the stock can be truncated. For an estimate of the optimal size of the buffer stock under such a regime, the method used in this paper requires some adaptation.

It can be added here that there is an important difference between the functions of national and international buffer stocks. The latter operate on the level of the world as a whole and cannot, therefore, be supported by imports. This is different for national buffer stocks. Their function is to allow, in combination with variations in import levels, a smooth absorption of variations in harvests, while the costs of running such a system are kept to a minimum.

\footnotetext{
${ }^{1}$ In the rest of this paper, $B$ indicates the desirable stock size.
} 


\section{CAUSES AND EFFECTS OF A DEPLETION OF STOCK}

The transient component of wheat production equals the difference between ne actual and the structural level of production $(V T=V-V S)$. So, in order to find $T$, we must first know the value of $V S$. The latter can be obtained from a function escribing the structural growth of wheat production. For Pakistan, such a function, ased on observations for the period from 1965-66 to $1983-84$, is $^{2}$

$$
\begin{aligned}
V S_{t}= & 4904.72 \times(1.050)^{t} \times 10^{3} \text { ton } \ldots \\
& (20.19) \quad(277.95) \\
& \bar{R}^{2}=0.92 ; \quad \text { D.W. }=1.42
\end{aligned}
$$

igures on wheat harvests before 1965-66 have been omitted because growth rates in at period differ significantly from those observed thereafter. It can further be dded that the functional form in eq. (4) appears to give slightly better results than ther forms involving the variable of time. No attempt has been made here to xplain the structural level of production as there was no need for that in the limited ontext of this paper. However, if an attempt is made to estimate optimum levels of heat stock in future years, it must at least be known whether the causes of this tructural growth will maintain their influence.

First, it can be noted in passing that, according to eq. (4), the growth rate of roduction surpassed the rate of population growth of about 3 percent by a considerble margin. This is a very important conclusion with far-reaching repercussions for he economy of Pakistan. But it should not divert us from the main purpose of eq. 4), which is to provide an indication of the structural level of wheat production and, hus, to allow derivation of the deviations from the growth path of structural producion for each year in the observed period. Expressed as percentages of the volume of tructural production, these deviations appear to fit very well in a normal distribu. ion. The mean value of the percentage deviations is -0.35 percent and the standard leviation is 10.04 percent. These simple findings propel the analysis a long way owards the estimation of the size of the wheat stock where costs are at their ninimum.

In the first place, they permit calculation of the probability that a negative leviation from the structural level of production overshoots a certain volume, or, $n$ other words, that a certain stock of wheat expressed as a percentage of the strucural level of production appears too small to compensate fully for a bad harvest. ${ }^{3}$

${ }^{2}$ Figures between brackets are $t$-values. Production volumes have been obtained from akistan Economic Survey, 1983-84 [3].

${ }^{3}$ The formulas applied for these calculations can be found, for example, in R. Hogg and A. raig [2].
This probability is represented by the expression $P(|x|>b)$, where $x$ indicates negative values of $V T$ as a percentage of $V S$ and $b$ is the initial stock as a percentage of VS. The values of this expression for different sizes of relative stocks are given in Column 2 of Table 1. For obvious reasons, they are lower for higher values of $b$. Secondly, the expected magnitude of production deviations in excess of certain given quantities can also be computed. In other words, for a given relative size of wheat stock it is possible to find the expected size of the negative percentage deviation from the structural level of production which surpasses this stock. Let us write this magnitude as $E(|x|>b)$. The values of this expression corresponding to different sizes of relative stocks are given in Column 3 of Table 1.

Table 1

$\mathrm{P}(|\mathrm{x}|>\mathrm{b})$ and $\mathrm{E}(|\mathrm{x}|>\mathrm{b})$ for Different Relative Sizes of Wheat Stocks

\begin{tabular}{ccc}
\hline $\begin{array}{c}\text { Size of Stock } \\
\text { as Percentage } \\
\text { of } V S\end{array}$ & $\begin{array}{c}P(|x|>b) \\
\text { in } \\
\text { Percentages }\end{array}$ & $\begin{array}{c}E(|x|>b) \\
\text { as } \\
\text { Percentage of } V S\end{array}$ \\
\hline$(1)$ & $(2)$ & $(3)$ \\
\hline 0 & 51.4 & -8.23 \\
2.5 & 41.5 & -9.43 \\
5.0 & 32.2 & -11.17 \\
7.5 & 23.8 & -13.05 \\
10.0 & 16.8 & -15.01 \\
12.5 & 11.3 & -17.02 \\
15.0 & 7.2 & -19.15 \\
17.5 & 4.4 & -21.12 \\
20.0 & 2.5 & -23.54 \\
\hline
\end{tabular}

Note: The underlying distribution of production deviations has been described in the text.

The significance of these findings can easily be seen. For example, it appears that the chance that a stock of 10 percent of structural production is wiped out in one year is 0.168 . The expected size of the corresponding negative deviation from the production trend is 15.01 percent of VS. The buffer stock absorbs 10 percent; so the expected volume of imports in this case is $0.168 \times 5.01$ percent of $V S=0.84$ percent of $V S$.

But this simple reasoning only applies in a one-year perspective. In a twoyear perspective several combinations of harvest results leading to supplementary import requirements must be considered. First, there is, of course, the case of a bad 
consistently lower than direct consumption estimates collected, at intervals, through, e.g., the Household Income and Expenditure Surveys.

If, for lack of alternative, wheat imports $(M)$ are regressed on transient production volumes $(V T)$ for the period from 1965-66 to 1981-82, the following result is obtained. ${ }^{4}$

$$
\begin{aligned}
M_{t+1}= & 826.81-0.73 \quad V T_{t} \\
& (8.39) \quad(-4.77) \\
& \bar{R}^{2}=0.59 ; \quad \text { D.W. }=1.27
\end{aligned}
$$

The Durbin-Watson statistic is just large enough to reject the null hypothesis. The egression coefficient is indeed negative, suggesting that imports of wheat have contributed significantly to the absorption of shocks caused by fluctuations of harvest volumes. It is important to note that an equation which includes a time variable to capture the development of the structural element of wheat imports performs less well than equation (6).

\section{RECURRENT COSTS OF A BUFFER STOCK SYSTEM}

The most important piece of information needed for calculating the recurrent costs of the buffer-stock system considered here, viz. the average annual volume of imports, is now available. In addition to this, the unit costs for storage and imports of wheat must also be known, but precise figures for these cost items do not seem to oe available. This is not very serious, however, as the calculations from here on are straightforward, such that the figures used below can be replaced, without further complications, by more accurate figures, when these are known. Still, it must be kept in mind that the magnitudes mentioned in the remaining part of this section are in the nature of approximations.

The unit costs of imports consist of three elements. The most important one is the difference between the purchase price paid in the international wheat market and the sale price obtained domestically, which tends to be much lower. An impression of the magnitude of this difference can be obtained by comparing average import prices of wheat in the past five years with wholesale prices. For this period the margin appears to vary mostly between 25 percent and 40 percent of the domestic prices. ${ }^{5}$ Another element to be considered is the undervaluation of foreign currency by the official exchange rate. The last and smallest element in the unit import costs consists of additional handling costs and the interest costs due to capital being locked up in the imported volume of wheat. Thus, by way of approximation, the total costs of wheat imports per volume unit are put here between 60 percent and 70 percent of the domestic price of wheat.
Three elements can also be distinguished in the costs of maintaining a stock of wheat. The first and most obvious element here relates to storage costs which consist of the rental costs of the storage facilities and the costs of handling. Then there are the interest costs in connection with the amount of capital invested in the stock. And, finally, there are the costs of annual buying and selling operations which result from the need to rotate the stock. The sum of these costs of maintaining a stock of wheat expressed per volume unit is estimated to be between 15 percent and 20 percent of the domestic price.

Before the storage costs corresponding to different sizes of desired stock can be estimated, it must be recalled that the actual average stock is larger than the desired stock, especially if the latter is small. This results from the fact that transient decreases of stock, in contrast with transient increases, are constrained by the nonnegativity of stocks (see Section 2). It can rather easily be seen that the difference between the actual average stock and the desired stock is precisely indicated by the average annual imports concurrent with the desired stock. This is because the latter variable represents the volume which, on average per year, is not detracted from the stock.

Finally, an assumption must be made regarding the time perspective adopted by the managers of the buffer stock. The figures in Table 2 show that the magnitudes of the expected annual imports can vary considerably with the view adopted in this regard. In the present exercise, a two-year perspective has been applied, but this is admittedly an arbitrary choice. Adoption of a different time span affects the outcomes of the calculations. The results obtained here are thus primarily of an illustrative nature.

The recurrent storage and import costs for different unit costs are presented in Table 3. As could be foreseen, storage costs rise and import costs fall as the desired stock size increases. As a result of this, total recurrent costs first drop and then rise if one moves to larger sizes of the desired stock. The particular stock size where total costs are the lowest depends, of course, on the relative importance of storage and import costs; the optimum size of stock is high, if storage costs are relatively low, and vice versa. This is illustrated by a comparison of the magnitudes in Columns 6 and 7 of Table 3 which shows that the former magnitudes reach their low at a lower level of the desired stock than that at which the latter magnitudes reach their low. Figure 1 illustrates this observation graphically. It suggests that, under the assumptions made in the exercise, the size of the wheat stock in Pakistan where the costs are at a minimum is between 2.5 percent and 7.5 percent of the level of structural production. In absolute terms, this corresponds to a wheat stock of between 300,000 and 900,000 tons in 1983 . 
Table 3

Estimates of Recurrent Costs in Percentages of the Value of Structural Wheat Production for Different Relative Sizes of Desired Stock and based on a Two-year Perspective

\begin{tabular}{|c|c|c|c|c|c|c|}
\hline \multirow{2}{*}{$\begin{array}{l}\text { Desired } \\
\text { Stock } \\
\text { Size as } \\
\% \text { of } V S\end{array}$} & \multicolumn{2}{|c|}{$\begin{array}{c}\text { Storage Costs, } \\
\text { if Unit Costs } \\
\text { are }\end{array}$} & \multicolumn{2}{|c|}{$\begin{array}{c}\text { Import Costs, } \\
\text { if Unit Costs } \\
\text { are }\end{array}$} & \multicolumn{2}{|c|}{$\begin{array}{c}\text { Total } \\
\text { Recurrent } \\
\text { Costs } \\
\end{array}$} \\
\hline & $\begin{array}{c}15 \% \text { of } \\
\text { the Domes- } \\
\text { tic Price }\end{array}$ & $\begin{array}{c}20 \% \text { of } \\
\text { the Domes- } \\
\text { tic Price }\end{array}$ & $\begin{array}{c}60 \% \text { of } \\
\text { the Domes- } \\
\text { tic Price }\end{array}$ & $\begin{array}{c}70 \% \text { of } \\
\text { the Domes- } \\
\text { tic Price }\end{array}$ & $\begin{array}{c}\text { Col. (2) + } \\
\text { Col. (5) }\end{array}$ & $\begin{array}{c}\text { Col. (3) }+ \\
\text { Col. (4) }\end{array}$ \\
\hline (1) & (2) & (3) & (4) & (5) & (6) & (7) \\
\hline 0 & 0.455 & 0.626 & 1.818 & 2.121 & 2.576 & 2.444 \\
\hline 2.5 & 0.740 & 0.966 & 1.458 & 1.701 & 2.441 & 2.424 \\
\hline 5.0 & 1.041 & 1.388 & 1.164 & 1.358 & 2.399 & 2.552 \\
\hline 7.5 & 1.343 & 1.790 & 0.870 & 1.015 & 2.358 & 2.660 \\
\hline 10.0 & 1.661 & 2.214 & 0.642 & 0.749 & 2.410 & 2.856 \\
\hline 12.5 & 1.991 & 2.654 & 0.462 & 0.539 & 2.530 & 3.116 \\
\hline 15.0 & 2.333 & 3.110 & 0.330 & 0.385 & 2.718 & 3.440 \\
\hline 17.5 & 2.681 & 3.574 & 0.222 & 0.259 & 2.940 & 3.796 \\
\hline 20.0 & 3.035 & 4.046 & 0.138 & 0.161 & 3.196 & 4.184 \\
\hline
\end{tabular}

recurrent costs

as $\%$ of value of

structural production

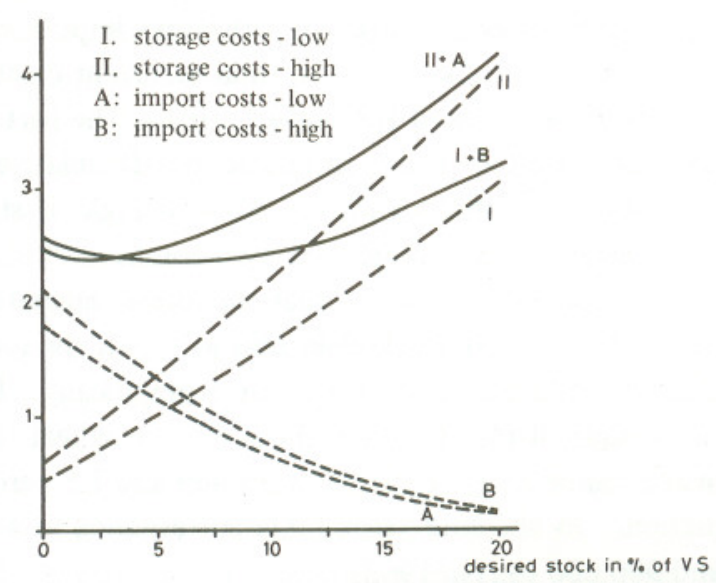

Figure 1: Recurrent Costs as a Function of the Desired Stock Size
So far it has been assumed that transient imports ${ }^{6}$ will commence only after the buffer stock has been exhausted completely. In practice, however, this rarely occurs. In order to avoid the suggestion of a crisis, supplementary imports are often arranged long before silos have emptied. The result is that a part of the buffer stock does not act as such and is, in fact, of a permanent nature. Thus, in reality the cutoff point beyond which stock decreases cannot go is not zero, but is situated at a positive volume of stock and the effective buffer stock is reduced accordingly. This affects both the actual average stock size and the average annual volume of imports, the former shifting upward and the latter shifting to the right. As a result, total recurrent costs increase for all sizes of the desired stock. However, if the exercise underlying the figures presented in Table 3 is repeated while maintaining a minimum stock of, for example, 2.5 percent of the level of structural production, the range of the stock sizes with the lowest levels of recurrent costs still remains between 2.5 percent and 7.5 percent of structural production.

\section{CONCLUDING REMARKS}

Under the assumptions made in the exercise presented in the foregoing section, the buffer stock of wheat in Pakistan which carries the lowest price tag appears to have been, in 1983, between 300,000 and 900,000 tons. Any magnitude within that range is only a fraction of the actual wheat stock of $2,100,000$ tons in that year. The question arises how this difference must be interpreted.

As indicated in the Introduction, buffer stocks, on the one hand, incur costs, but, on the other hand, provide security. A yardstick by which this security can be measured in quantitative terms does not exist, but it can safely be assumed that it varies inversely with the volume of imports required to supplement the buffer stock. Thus, it can reasonably be argued that security rises with the size of the buffer stock. From this, it follows immediately that any size of buffer stock smaller than the stock where the costs reach a minimum cannot be optimal, as such a stock would have negative marginal costs and a positive marginal benefit (security). It is certainly possible, however, that the optimal size of the buffer stock is larger than the minimum-cost stock size. The precise position of the optimum depends, of course, on a comparison of marginal costs and benefits.

The reasoning followed in the preceding paragraph refers specifically to the desirable size of the buffer stock, i.e. the size of stock that is to be reached on average, after allowing for the truncation due to non-negativity of the stock. Actual stocks can - and will - differ from the optimum; in fact, such differences are wholly in accordance with the function of the buffer stocks. In this connection, it is worth while recalling that the 1983 wheat stock was affected favourably by good harvests in the previous years.

${ }^{6}$ This paper has concentrated on transient imports, next to other variables. Note, however, that the total wheat import bill also consists of structural imports or exports of wheat. 
In conclusion, it must be pointed out that the link between the size of the buffer stock and the magnitude of structural imports (or exports) has not been examined. This is fully justified in the present case, because structural production of wheat, according to equation (4), and domestic consumption are of the same order of magnitude. Therefore, structural imports or exports of wheat can, at present, be ignored. But in other cases, where structural imports or exports occur in significant volumes, their relation with the desirable stock size must also be considered.

Suppose for a moment that Pakistan produces considerably more than it consumes and that the difference is exported. In this case, a bad harvest simply means that the exportable surplus would be reduced temporarily. But, disasters aside, supplementary imports are not required. It implies that the argument of the costly imports - which favours the maintenance of a buffer stock - does not apply. In fact, the stock is to be kept as low as possible in this case, because it pays to profit from the higher world-market price by maximizing the export volume.

Inversely, if Pakistan should be a structural importer, wheat must be obtained from abroad even in years of relatively good domestic harvests. A buffer-stock system cannot avoid this pattern and, therefore, does not make sense. Of course, even in this case, a stock is to be maintained in order to guarantee a smooth supply of wheat over time. But such a stock does not act as a buffer absorbing the variations in yearly domestic production. It appears, therefore, that the issue of the optimal size of the buffer stock of wheat is of particular relevance in Pakistan now that a structural balance seems to exist between domestic production and consumption.

\section{REFERENCES}

Cornelisse, Peter A., and Syed Nawab Haider Naqvi. The Anatomy of the Wheat Market in Pakistan. Rotterdam: Erasmus University/Islamabad: Pakistan Institute of Development Economics. October 1984.

Hogg, R., and A. Craig. Introduction to Mathematical Statistics. New York: MacMillan. 1959.

Pakistan. Finance Division. Economic Adviser's Wing. Pakistan Economic Survey, 1983-84. Islamabad. 1984.

Pakistan. Ministry of Food, Agriculture and Co-operatives. Food and Agriculture Division. Agricultural Statistics of Pakistan, 1982. Islamabad. 1983.

\section{Comments on \\ "On the Optimal Size of a Buffer Stock - The Case of Wheat in Pakistan"}

The failure of wheat crop in Pakistan for two successive years (1983-84 and 1984-85) has turned a state of comfortable self-sufficiency into an uncomfortable deficit. It has, in fact, wiped out a stock of over 2.0 million metric tons that existed in the country in early 1983 . Food security has again become a serious issue and the cost of imported wheat adds another burden to the economy.

The question of the "optimum" size of a buffer stock has assumed a new urgency. Cornelisse and Kuijpers should be congratulated on making this attempt which is incidentally the first ever made in Pakistan - to analyse the issues of security and cost involved in maintaining a buffer stock in the face of unstable production of wheat from year to year. It is, however, important to note that, as the authors themselves admit, it is only a "stepping stone" to a decision on the optimal size of the stock of wheat in Pakistan. May I add that this exercise is an important stepping stone in that direction. Let me turn to a few interesting questions that the paper raises.

Since the size of the buffer stock depends on the volume of "transient" production (Equation 3), it is important to understand what this production itself depends on. Transient production is the difference between "actual" and "structural" levels of production. What are the factors that determine the structural level of production? This is never made clear by the authors, although it is perhaps the most important question in determining the transient level and hence the probability of the stock matching this level. Is structural growth simply the level of production that excludes the time trend? If structural production means "normal" production, then the authors should have made an attempt to determine the "good" and "bad" years.

The next problem is that of integrating a multi-year perspective into the system of probabilities. What are the combinations of the expected "bad" and "good" harvests over three years that seem reasonable? I think a way around the arbitrary scenarios developed by the authors in this exercise would be to estimate the expected harvests by a forecasting model. This would reduce (a) the degree of arbitrariness and (b) the number of reasonable scenarios for two to three years. 
I think there is no reason not to use the data of the 1979 Household Income and Expenditure Survey to derive independent estimates of wheat consumption or "structural imports". Some elasticity estimates already exist. Growth rates for population and per capita income are also available. Equation 6 is dubious in this respect, because it says nothing about consumption: it repeats what is obvious (negative sign of the coefficient).

It is somewhat surprising that the unit cost of the imported wheat in Pakistan is only 6070 percent of the domestic price of wheat. Other studies have shown that the domestic selling price of wheat has been consistently lower than the imported price, even if the foreign exchange is corrected for undervaluation. How does one really determine the equivalence of the international and domestic prices of wheat? Should we not really compare its border price with the domestic resource cost? Maybe, we should not even worry about the international price, because the basic aim is to be "self-sufficient" in wheat.

A crucial consideration in maintaining a buffer stock is its cost, including storage, transportation and handling charges. How have the authors estimated that this cost would be $10-20$ percent of the domestic price of wheat? Since recurrent cost is an important component of a positive (permanent) stock, how close should the levels of actual and desired stocks be? The numerical data in the exercise at the end show that two successive crop failures have exhausted a stock of 2.0 million metric tons, which was nearly two-thirds as high as the upper limit of the "desired" level of stock set by the authors at a minimum recurrent cost !

What is the level of stock that provides minimum security and can be maintained at a minimum cost? I hope that the authors will expand their basic framework to determine the optimum level of a buffer stock, answering the questions of security and cost (of imports and maintenance of stock). This exercise can indeed develop into a useful policy framework for regulating wheat stocks. In any case, it will have advanced the state of the art in Pakistan.

\section{Size - Productivity Relationship in Pakistan's Agriculture in the Seventies}

\author{
M. GhaFFAR ChaUdhry, ManzoOR A. Gill \\ and GHUlam MUSTAFA CHAUdHRY*
}

A large majority of the less developed countries, including Pakistan, suffer from low levels of productivity in agriculture. In view of the scarcity of farm land and the limitations of intensive margins of cultivation, the importance of raising these levels may not be underestimated in terms of a more judicious use of labour force, rapid growth of agricultural production and increased availability of funds for industrial development. Productivity gains favouring well-to-do farmers, however, may produce undesirable results such as deterioration of income inequalities, political instability and social unrest, and must be avoided as far as possible. It is in this context that the distribution of productivity gains among various size groups should be taken to be as important as the aggregate growth of farm productivity, if not more.

In spite of the discontinuities at times, aggregate productivity in Pakistan's agriculture has been on the increase since the early Sixties. While the distribution of these gains is a controversial issue, it has implications for the fundamental size productivity relationship. For example, it is a commonly accepted view that the productivity increases of the Sixties were largely concentrated in the hands of the large farmers because of their financial superiority for investing in key agricultural inputs and high-value cash crops and their advantageous position for benefiting from government programmes of input subsidies, price supports and institutional credit [7, p. $706 ; 8$, p. 364; and 19, p. 196]. Arguing on these lines, Berry and Cline have statistically shown, although on the basis of comparisons of inherently incomparable data, that in Pakistan the large farmers have overtaken the traditionally more productive small farmers in productivity [2, pp. 90-125]. Mahmood and Nadeem [11, p. 186], however, deny the existence of a positive size - productivity relationship on the basis of the 1973 farm data and argue that the smallest and the largest farms have the highest productivity. By contrast, a recent study by one of the authors of this paper concluded that the rate of growth of the productivity of the small farmers

*The authors are, respectively, Chief of Research, Associate Staff Economist and Technical Assistant at the Pakistan Institu te of Development Economics, Islamabad. 